Article

\title{
Molecular Cloning and Functional Characterization of Tibetan Porcine STING
}

\author{
Zhiqing Huang, Xiaoling Chen, Keying Zhang, Bing Yu, Xiangbing Mao, Ye Zhao \\ and Daiwen Chen *
}

Key Laboratory for Animal Disease-Resistance Nutrition of Sichuan Province, Institute of Animal Nutrition, Sichuan Agricultural University, Yaan, Sichuan 625014, China;

E-Mails: zqhuang@sicau.edu.cn (Z.H.); xlchen@ sicau.edu.cn (X.C.); zkeying@yahoo.com (K.Z.); bingyucn@yahoo.com (B.Y.); xiangbingm@hotmail.com (X.M.); ye_zhao@yahoo.com (Y.Z.)

* Author to whom correspondence should be addressed; E-Mail: dwchen@ @icau.edu.cn; Tel.: +86-835-288-2088; Fax: +86-835-288-2088.

Received: 30 November 2011; in revised form: 22 December 2011 / Accepted: 22 December 2011 / Published: 4 January 2012

\begin{abstract}
Tibetan pig is well known for its strong disease resistance. However, little is known about the molecular basis of its strong resistance to disease. Stimulator of interferon (IFN) genes (STING), also known as MPYS/MITA/ERIS/TMEM173, is an adaptor that functions downstream of RIG-I and MAVS and upstream of TBK1 and plays a critical role in type I IFN induction. Here we report the first cloning and characterization of STING gene from Tibetan pig. The entire open reading frame (ORF) of the Tibetan porcine STING is $1137 \mathrm{bp}$, with a higher degree of sequence similarity with Landrace pig (98\%) and cattle $(88 \%)$ than with chimpanzee $(84 \%)$, human $(83 \%)$ or mouse $(77 \%)$. The predicted protein is composed of 378 amino acids and has 4 putative transmembrane domains. Real-time quantitative PCR analysis indicated that Tibetan pig STING mRNA was most abundant in the lung and heart. Overexpression of Tibetan porcine STING led to upregulation of IFN- $\beta$ and IFN-stimulated gene 15 (ISG15) in porcine jejunal epithelial cell line IPEC-J2 cells. This is the first study investigating the biological role of STING in intestinal epithelial cells, which lays a foundation for the further study of STING in intestinal innate immunity.
\end{abstract}

Keywords: cloning; innate immunity; IPEC-J2 cells; Tibetan porcine STING; type I interferon 


\section{Introduction}

Innate immunity is the first line of host defense against invading pathogens. Innate immune responses are initiated by the host's pattern recognition receptors (PRRs), which recognize conserved pathogen-associated molecular patterns (PAMPs) of microorganisms [1-3]. Upon recognition, PRRs trigger a series of signaling events leading to the expression of type I interferons (IFNs), IFN-stimulated genes (ISGs) and proinflammatory cytokines, which are all critical for the protection of a host suffering from microbial infection [4]. The host has developed at least two distinct mechanisms for the recognition of viral RNAs, which represents the first step of innate antiviral response [5]. One is mediated by Toll-like receptors (TLRs), such as TLR3, which recognizes viral double-stranded RNA (dsRNA) released by infected cells [6]. The other mechanism involves the RNA helicases RIG-I and MDA5, which function as intracellular viral RNA sensors [7,8].

Stimulator of IFN genes (STING), also known as TMEM173/MPYS/MITA/ERIS, has recently been shown to be an adaptor that functions downstream of RIG-I and MAVS and upstream of TBK1 $[9,10]$. Overexpression of STING activates interferon regulatory factor 3 (IRF3), leads to upregulation of type I IFNs as well as ISGs expression, and inhibits viral replication, whereas knockdown of endogenous STING has the opposite effects [11-13]. These results demonstrate that STING is a key component in the virus-triggered IRF3 activation pathway and cellular antiviral response and plays a critical role in type I IFN induction.

Tibetan pig is a special Chinese indigenous pig breed, which is distributed in the Qinghai-Tibet Plateau at altitudes ranging from 2200 to $4400 \mathrm{~m}$. It is well known for its remarkably strong disease resistance compared to other pig breeds. In this study, we cloned the Tibetan porcine STING cDNA and examined its tissue distribution. We also examined the effects of STING on IFN- $\beta$ and ISG15 expressions at mRNA levels.

\section{Results and Discussion}

\subsection{Cloning and Sequence Analysis of Tibetan Porcine STING}

Based on the sequence of Landrace pig STING (GenBank ID: FJ455509), the primers pMD19-T-STING-F and pMD19-T-STING-R were designed and used to amplify the potential STING cDNA sequence from total RNA extracted from skeletal muscle of Tibetan pigs. The entire open reading frame (ORF) of Tibetan porcine STING contains 1137 bp (GenBank ID: JN226147) and encodes 378 amino acid residues (Figure S1). This nucleotide sequence shares 77\%, 83\%, 84\%, and $88 \%$ homology with the known STING sequences of mouse (GenBank ID: NM_028261), human (GenBank ID: NM_198282), chimpanzee (GenBank ID: XM_001135484), and cattle (GenBank ID: NM_001046357), respectively. Not surprisingly, the nucleotide sequence of STING in the Tibetan pig is nearly identical to that of Landrace pig reported by Xie et al. [14], with 12 nucleotide differences. Whether some of these 12 differences may be due to single nucleotide polymorphisms need to be identified in future investigations. 
Figure 1. Alignment, phylogenetic tree, and architecture of the predicted amino acid sequence for Tibetan porcine STING. (A) Multiple comparison of amino acid sequences of Tibetan pig, Landrace pig, chimpanzee, cattle, human and mouse STING. Four putative transmembrane regions (TM) of Tibetan porcine STING are indicated by bold lines. The numbers on the right of every line refer to positions of the amino acid residues; (B) Phylogenetic analysis of Tibetan porcine STING. The neighbor-joining tree was constructed by DNAMAN. The sequences were derived from the predicted amino acid sequences of Tibetan porcine STING (AEL97644) and the GenBank entries with accession numbers ACJ70708 (Landrace pig), NP_001039822 (cattle), XP_001135484 (chimpanzee), NP_938023 (human), and NP_082537 (mouse). The scale bar is 0.05 .

Tibetan pig Landrace pig Cattle

Chimpanzee

Human

Mouse

Tibetan pig Landrace pig Cattle

Chimpanzee

Human

Mouse

Tibetan pig Landrace pig Cattle

Chimpanzee

Hurnan

Mouse

Tibetan pig

Landrace pig

Cattle

Chimpanzee

Human

Mouse

Tibetan pig

Landrace pig

Cattle

Chimpanzee

Human

Mouse

Tibetan pig Landrace pig Cattle Chimpanzee

Human

Mouse

Tibetan pig

Landrace pig

Cattle

Chimpanzee

Human

Mouse
TM1

MPYSSLHPS IPQPRGLR AQE A ALVLLGACLVALWGLGELPE YTLRWLVL HL ASQQIGLLV MPYSSLHPS IPQPRGLRAQVAALVLLGACLVALWGLGELPEYTLRWLVLHL ASQQIGLLV MP HSSL HPS IPQP RGL RAQKA ALVLLSACLVALWGLGE P P YTL KWLVL HL ASQQMGLL I MPHSSL HPS IPCPRGHG AQKA ALVLLS ACLVTL WGLGEP PE HTL RYLVL HL ASLQLGLLL MPHSSLHPS IPCPRGHGAOKAALVLLS ACLVTL WGL GEPPEHTLRYLVL HL ASLQLGLLL MPYSNL HP A IPRP RGHRSKYVAL IF LVASLMIL WVAKDP PNHTLKYL AL HL ASSHELGLLL \#夫 TM2

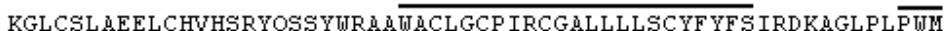
KGLCSL ДEELCHVHSRYQSSYWRÄAR ACLGCP IRCGALLLLSCYF YF SIRDKAGLPLPWM KGICSL AEELCHVHSRYHGSYWRAVRACLCSSMRCGALLLLSCYF YCSLPNMADLPF TWM NGVCSL AEELRH IHSRYUGSYURTVRACLGCPLRRG ALLLLS I YFYYSLPNAVGPPFTWM NGVCSLAEELRH IHSRYRGSYWRTVRACLGCPLRRGALLLLS IYFYYSLPNAVGPPFTWM KNLCCLAEELCHVQSRYQGSYUKAVRACLGCP IHCMAM ILLSSYF YF-LQNT AD I YLSWM

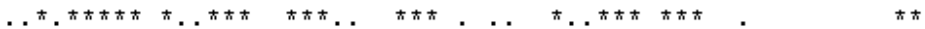
TM3 TM4

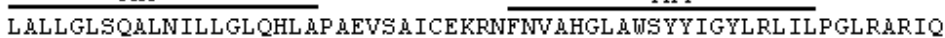
LALLLGLSQALN ILLGLQHL ÀP AEVS A ICE KRNFNV AHGL ẢSYY I GYLRL IL PGLRAR IQ LALLGLSQALN ILL GLQGL AP AEVS A ICE KRNFNV A HGL A WSYY I GYLRL IL PGL P AR IQ L AL LGLSQALN IL LGL KGL AP AE IS AVCE KGNFNV AHGL A TSSYY I GYL RL IL PELQAR IR LALLLGLSQALN ILLGL KGL AP AE IS AVCE KGNFNV A HGL ÄWSYY IGYLRL IL PELQAR IR FGLLVL YKSLSMLLGLQSLTP AEVS AVCEE KKLNV AHGL A WSYY IGYLRL IL PGLQAR IR

ネ夫

A YNQRHKNVLGG IGNHRL HILF PLDCG APDDLSV ADPNIRF L HKL PQQS ADR A G IKGRVY A YNQRHKNVLGG IGNHRL H ILF PLDCGVPDDLSV ADPN IRF L HE L PQQS ADR A G IKGRVY I YNQF HNNTLQGA GSHRL H IL F PLDCGVPDDLNV AD PN IRF L HEL PQQS AD RA G IKGRVY TYNOHYNNLLRG BVSORL Y ILLPLDCGVPDNLSM \&DPNIRFLDKLPOOT LDR AG IKDRVY TYNQHYNNLLRGAVSQRL Y ILL PLDCGVP DNLSMẢD PN IRF LDKL PQQTGDHÄGI KDRVY MFNQL HNNMLSGAGSRRL Y ILF PLDCGVPDNLSVVD PN IRF RD ML PQQN IDRAGI KNRVY 草

TNS I YELLENGQP A GVCVLEY Y TPLQTLF AMSQDGR AGFSREDRLEQAKLFCRTLED IL A TNS I YEL L ENGQP A GVCVL GY A TPLQTLF AMSQDGR A GFSREDRLEQAKLFCRTLED IL TNS I YELLENGQR A GVCVLEY ATPLQTLF AMSQDGR A GF SREDRLEQAKLFCRTLED IL A SNS I YELLENGQR AGTCVLEY ATPLQTLF AMSQYSQAGFSREDRLEQAKLFCRTLED IL A SNS I YELLENGQRA GTCVLE Y A TPLQTLF AMSQYSQA GF SREDRLEQAKLFCRTLED ILA SNSVYE ILENGQP AGVC ILE YẢTPLQTLF AMSQD AKA GF SREDRLEQAKLFCRTLEE ILE

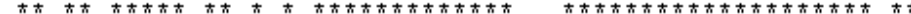

DAPE AONNCRL IVYQE PTEGGSF SLSQE ILRHLRQEERE-VTMGS AE TSVVPTSSTLSQE DAPE AONNCRL IVYOEP TEGGSF SLSOE ILRHL ROEERE-VTMGS AE TSVVPTSSTLSOE NAPESQNNCRL IVYQE $P$ AE GSSF SLSQE ILQHLRQE ERE-VTMGSTE TSVMP GSSVLSQE DAPESONNCRL I A YQEP ADDSSF SLSQEVLRHLRQEE KEEVTVGSLKTS AVPSTSTMSQE DAPESQNNCRL I A YQE P ADDSSF SLSQEVL RHLRQEE KEEVTVGSLKTS AVPSTSTMSQE DVPESRNNCRL IVYQEP TD GNSF SLSQEVLRH IRQE E KE EVTMNAPMTSVAPPPSVLSQE

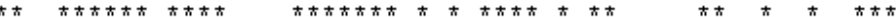


Figure 1. Cont.

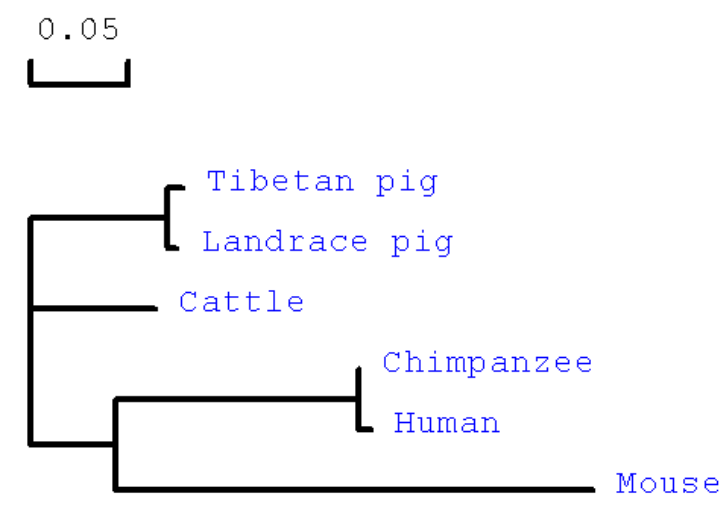

(B)

The predicted amino acid sequence of the Tibetan porcine STING was compared with mammalian STING amino acid sequences available from GenBank (Figure 1A). There was 69\%, 76\%, 77\% and 86\% similarity to that of mouse (NP_082537), human (NP_938023), chimpanzee (XP_001135484) and cattle STING (NP_001039822), respectively. Not surprisingly, the Tibetan porcine STING protein sequence (AEL97644) had 98.68\% homology with known Landrace pig sequence (ACJ70708), with 5 amino acid substitutions at residues 20 (Glu-Val), 86 (Trp-Arg), 208 (Ala-Val), 224 (Lys-Glu) and 260 (Glu-Gly). Structural analysis with the SMART program (http://smart.embl-heidelberg.de/smart/set_ mode.cgi?NORMAL=1) showed that Tibetan porcine STING contains four putative transmembrane (TM) domains (aa 15-37, aa 86-108, aa 118-140, and aa 153-172) at its N-terminus (Figure 1A). Similar transmembrane domains have also been reported in the STING sequences of human [13] and Landrace pig [14] and can be predicted in the mouse STING sequence, suggesting that transmembrane domains may be the common features of mammalian STING. In addition, the transmembrane domains of human STING have been demonstrated to be critical to retain this protein on the membrane of endoplasmic reticulum or mitochondria $[12,13]$. As mentioned above, there were 5 amino acid differences between Landrace and Tibetan porcine STINGs. Among them, an amino acid substitution at residues 20 (Glu-Val) was observed in its transmembrane domains. Whether this substitution may lead to the differences in disease resistance between Landrace and Tibetan pigs need further investigation. A phylogenetic analysis of the amino acid sequence was performed, and the resulting neighbor-joining tree showed that the Tibetan pig has a closer genetic relationship with Landrace pig and cattle than with chimpanzee, human and mouse (Figure 1B).

\subsection{Tissue Distribution of the Tibetan Porcine STING $m R N A$}

The mRNA expression profile of Tibetan porcine STING was determined by real-time quantitative PCR in various tissues. As shown in Figure 2, Tibetan porcine STING transcripts were most abundant in the lung and heart, followed by the skeletal muscle, thymus, fat, small intestine, lymph node and liver, and to a lesser extent in the spleen and kindey. The tissue distribution pattern of STING has been previously examined in several mammals, including human [11,13], mouse [12] and Landrace pig [14]. Landrace pig STING mRNA was mainly expressed in the spleen, lymph node and lung [14], while Tibetan pig STING mRNA was mainly detected in the lung and heart in the present study. The 
reason for this discrepancy may be due to the different age and health status of the animals. In addition to the lung and heart, Tibetan porcine STING was also detected in all other examined tissues, indicating that Tibetan porcine STING may be important in the innate immune system in various organs of the pigs.

Figure 2. Relative mRNA expression of Tibetan porcine STING in different tissues. Total RNA from different tissues of 3 healthy Tibetan pigs was used to perform the real-time quantitative PCR. Samples were performed in duplicate. The amount of Tibetan porcine STING mRNA was normalized to the amount of $\beta$-actin mRNA. Data are presented as mean $\pm \mathrm{SE}(n=3)$, in arbitrary units.

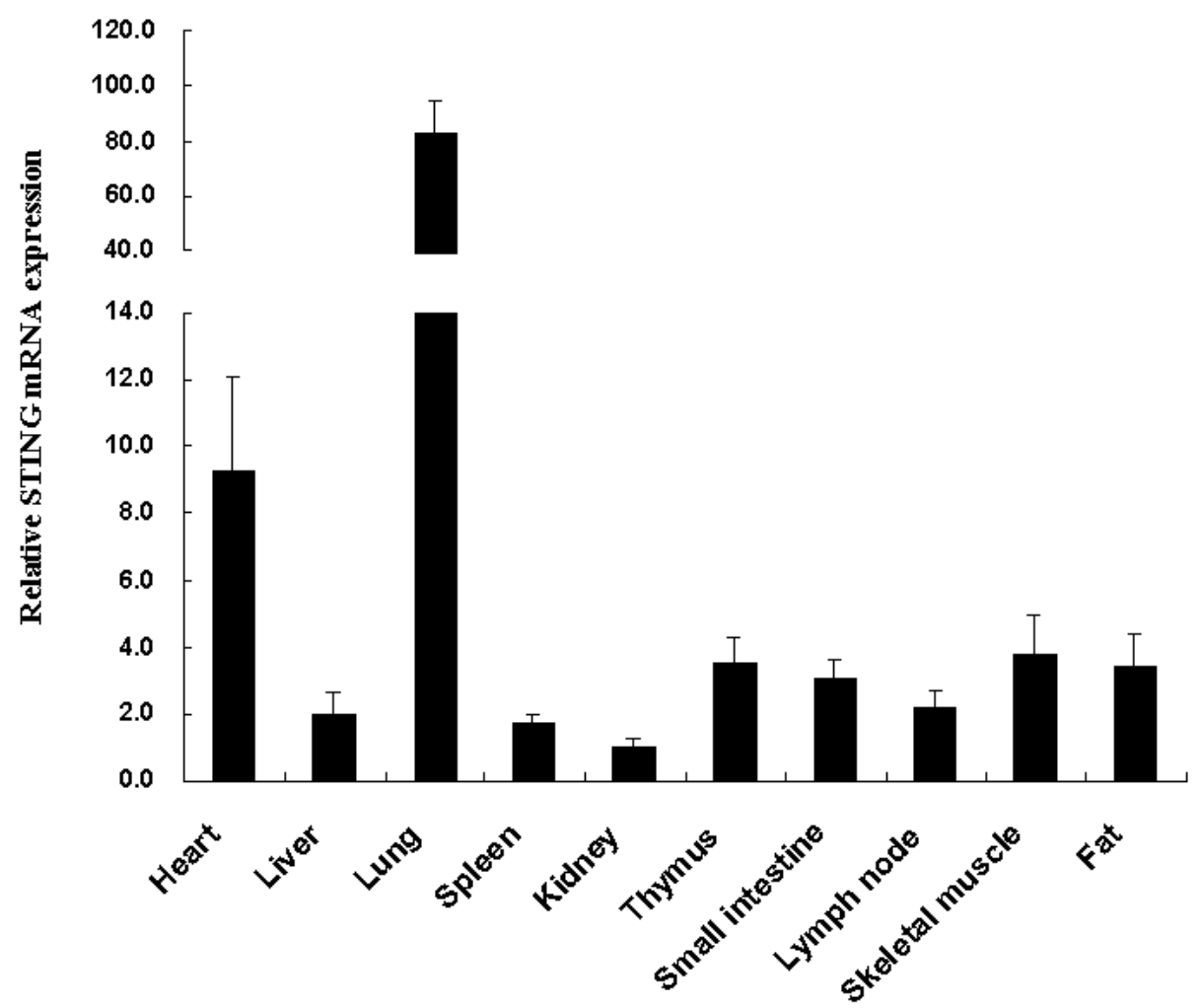

\subsection{Overexpression of Tibetan Porcine STING Leads to Upregulation of IFN- $\beta$ and ISG15} in IPEC-J2 Cells

An inconceivable number of microorganisms live in the intestinal tract of animals and humans [15]. Intestinal epithelial cells participate in the onset and regulation of the intestinal innate immune response to enteric virus infection [16]. During viral infection, IFN- $\alpha$ and IFN- $\beta$ protect cells against viral invasion by interfering with viral replication. Although STING has been well-characterized as an IFN stimulator since it was cloned in 2008 [11-14], there is no report of its function on intestinal epithelial cells. To examine whether Tibetan porcine STING is capable of stimulating expression of IFN- $\beta$ in the porcine jejunal epithelial cell line IPEC-J2 cells, the Tibetan porcine STING expression plasmid was constructed and transfected into IPEC-J2 cells. Twenty-four hours after the transfection, the mRNA level of Tibetan porcine STING in IPEC-J2 cells transfected with the plasmid pcDNA3.1(+)-STING increased $443.56 \pm 70.35$ times $(n=3)$, compared with cells transfected with the empty vector pcDNA3.1 $(+)$ control (data not shown). Thus, the mRNA level of IFN- $\beta$ was tested. As shown in Figure 3, IFN- $\beta$ 
was significantly upregulated by Tibetan porcine STING overexpression. ISG15 (an IFN-stimulated gene) is strongly upregulated by type I IFN [17]. Here, we showed that ISG15 was also significantly up-regulated by overexpression of Tibetan porcine STING in IPEC-J2 cells (Figure 3). This is the first study investigating the biological role of STING in intestinal epithelial cells, which lays a foundation for the further study of STING in intestinal innate immunity.

Figure 3. Overexpression of Tibetan porcine STING leads to upregulation of IFN- $\beta$ and ISG15. IPEC-J2 cells were transfected with $2 \mu \mathrm{g} /$ well of the plasmid pcDNA3.1(+)-STING or the empty vector pcDNA3.1(+). IFN- $\beta$ and ISG15 mRNA levels were determined using real-time quantitative PCR $24 \mathrm{~h}$ after transfection. The amount of IFN- $\beta$ and ISGI5 mRNA was normalized to the amount of $\beta$-actin mRNA. Results are the mean and standard errors from three independent experiments performed in duplicate. $* P<0.05$ and $* * * P<0.001$ as compared with empty vector.

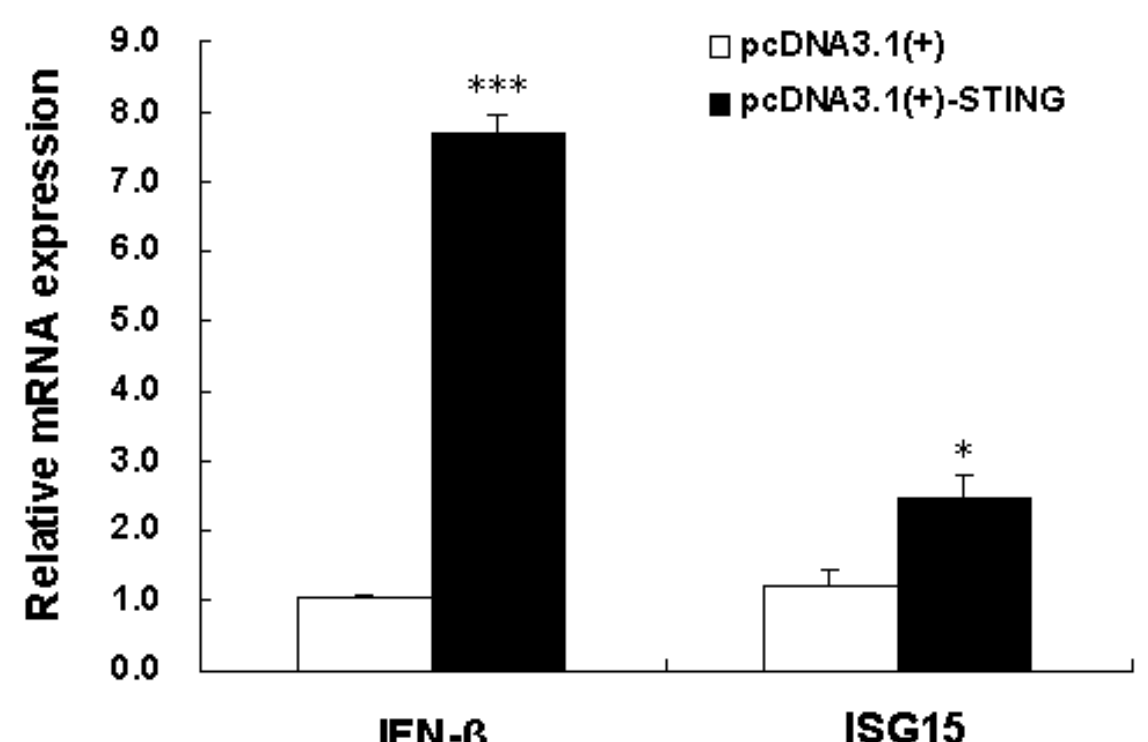

\section{Materials and Methods}

\subsection{Animals and Tissue Sample Collection}

Three Tibetan female pigs (body weight of $15.47 \pm 0.41 \mathrm{~kg}$ ) were slaughtered by exsanguination according to protocols approved by the Animal Care Advisory Committee of Sichuan Agricultural University. The heart, liver, lung, spleen, kidney, thymus, small intestine (jejunum), lymph node, skeletal muscle and fat were removed and immediately snap frozen in liquid nitrogen before being stored at $-80{ }^{\circ} \mathrm{C}$ for RNA isolation.

\subsection{RNA Isolation and Reverse Transcription}

Total RNA was extracted from collected tissue samples using RNAiso Plus reagent (TaKaRa, Dalian, China) according to the manufacturer's instructions. The concentrations of total RNA were determined spectrophotometrically using a Beckman Coulter DU800 (Beckman Coulter, Fullerton, CA, USA). One microgram of total RNA from each sample was reverse transcribed in a final volume 
of $20 \mu \mathrm{L}$ using a PrimeScript ${ }^{\circledR}$ RT reagent Kit with gDNA Eraser (TaKaRa) according to the manufacturer's protocols. The first-strand cDNA was subsequently used as a template for PCR.

\subsection{Cloning of STING cDNA}

The full coding region of STING was obtained by PCR amplification from the total RNA extracted from skeletal muscle of Tibetan pigs. A pair of primers (pMD19-T-STING-F and pMD19-T-STING-R) were designed based on the sequence of Landrace pig STING (GenBank ID: FJ455509) and were as follows: 5'-ATGCCCTACTCCAGCCTGCATC-3' (forward) and 5'-TCAGAAGATATCTGAGCGG AG-3' (reverse). The PCR was performed in a $50 \mu \mathrm{L}$ reaction volume containing $2 \mu \mathrm{L}$ of the first-strand cDNA, $1 \mu \mathrm{L}$ each of forward and reverse primers from $10 \mu \mathrm{M}$ stocks, $21 \mu \mathrm{L}$ DEPC-treated water, and $25 \mu \mathrm{L}$ of $2 \times$ Taq PCR MasterMix (Tiangen, Beijing, China). The thermal cycling conditions used were: 1 cycle of $94{ }^{\circ} \mathrm{C}$ for $3 \mathrm{~min}$, then 35 cycles of $94{ }^{\circ} \mathrm{C}$ for $30 \mathrm{~s}, 63{ }^{\circ} \mathrm{C}$ for $30 \mathrm{~s}$, and $72{ }^{\circ} \mathrm{C}$ for $1 \mathrm{~min} 30 \mathrm{~s}$, and followed by 1 cycle of $72{ }^{\circ} \mathrm{C}$ for $7 \mathrm{~min}$. The PCR product was purified, cloned into pMD19-T vector (TaKaRa) and sequenced, resulting in pMD19-T-STING.

\subsection{Plasmid Construction}

The DNA fragment containing the entire open reading frame (ORF) of Tibetan porcine STING was PCR amplified using the specific primers (pcDNA3.1(+)-STING-F: 5'-TACGAATTCATGCCCTACT CCAGC-3' and pcDNA3.1(+)-STING-R: 5'-GCTCTAGATCAGAAGATATCTGAGCG-3') and the plasmid pMD19-T-STING as a template. Primer pcDNA3.1(+)-STING-F introduced an EcoRI site, and primer pcDNA3.1(+)-STING-R contained an XbaI site (underlined). After digestion with EcoRI and $X b a \mathrm{I}$, the PCR product was inserted into the vector pcDNA3.1(+) (Invitrogen, Carlsbad, CA, USA). Proper construction was confirmed by sequencing and was designated as pcDNA3.1(+)-STING.

\subsection{Cell Culture}

The IPEC-J2 cell line, originally derived from jejunal epithelia of a neonatal unsuckled piglet [18], was a kind gift from Dr. Junjun Wang (College of Animal Science and Technology, China Agricultural University, Beijing, China). IPEC-J2 cells were maintained in Dulbecco modified Eagle medium (DMEM)/Ham's F-12 (1:1) medium (Invitrogen) supplemented with 10\% fetal bovine serum (FBS) (Invitrogen), and antibiotics (100 U/mL penicillin and $100 \mu \mathrm{g} / \mathrm{L}$ streptomycin) (Invitrogen) at $37{ }^{\circ} \mathrm{C}$, $5 \% \mathrm{CO}_{2}$ in a humidified atmosphere. Medium was renewed every 2 days.

\subsection{Transfection}

IPEC-J2 cells were seeded in 6-well plates (Corning, NY, USA) at a density of $2.0 \times 10^{5}$ cells/well $24 \mathrm{~h}$ prior to transfection. Two micrograms of the plasmid pcDNA3.1(+)-STING or an equal amount of the empty vector pcDNA3.1(+) were transfected using Lipofectamine 2000 (Invitrogen). At $24 \mathrm{~h}$ post-transfection, total RNA was extracted and reverse transcribed according to the above-mentioned method. All plasmids were prepared with Endo-free plasmid kit (Omega, Norcross, GA, USA) following the manufacture's instruction. 


\subsection{Real-Time Quantitative PCR}

Real-time quantitative PCR was performed in a CFX96 Real-Time PCR Detection System (Bio-Rad, Hercules, CA, USA). The gene-specific primers used are listed in Table 1. The PCR mixture consisted of $2 \mu \mathrm{L}$ of the first-strand cDNA sample, $1 \mu \mathrm{L}$ each of forward and reverse primers from $10 \mu \mathrm{M}$ stocks, $6 \mu \mathrm{L}$ DEPC-treated water, and $10 \mu \mathrm{L}$ of $2 \times$ SsoFast EvaGreen Supermix (Bio-Rad). The PCR cycling conditions used were: 45 cycles of $98{ }^{\circ} \mathrm{C}$ for $2 \mathrm{~s}$ and $60{ }^{\circ} \mathrm{C}$ for $5 \mathrm{~s}$. Each primer pair used yielded a single peak in the melting curve and a single band with the expected size in agarose gel. Identities of the PCR products were confirmed by sequencing. Data were analyzed according to the efficiency-corrected comparative $\mathrm{Ct}$ method [19] and were normalized by $\beta$-actin expression in each sample.

Table 1. Primers used in real-time quantitative PCR.

\begin{tabular}{|c|c|c|c|c|}
\hline Gene & Primer & Sequence & GenBank ID & Product size \\
\hline \multirow[t]{2}{*}{$I F N-\beta$} & Forward & 5'-AAATCGCTCTCCTGATGTGT-3' & NM 001003923 & $78 \mathrm{bp}$ \\
\hline & Reverse & 5'-TGCTCCTTTGTTGGTATCG-3' & & \\
\hline \multirow[t]{2}{*}{$I S G 15$} & Forward & 5'-AGCAACGCCTATGAGGTC-3' & EU584557 & $101 \mathrm{bp}$ \\
\hline & Reverse & 5'-AAAGTCAGCCAGAAATGGTC-3' & & \\
\hline \multirow[t]{2}{*}{$\beta$-actin } & Forward & 5'-CCACGAAACTACCTTCAACTCC-3' & $\underline{\text { DQ845171 }}$ & $132 \mathrm{bp}$ \\
\hline & Reverse & 5'-GTGATCTCCTTCTGCATCCTGT-3' & & \\
\hline
\end{tabular}

\subsection{Statistical Analysis}

Data were expressed as mean \pm SE. One-way ANOVA and Tukey's tests (SPSS Inc., Chicago, IL, USA) were performed to assess the statistical significance between treatments. Statistical significance was set at $P<0.05$.

\section{Conclusions}

Tibetan pig is well known for its strong disease resistance. However, little is known about the molecular basis of its strong resistance to disease. In this study, we first cloned Tibetan porcine STING, which encodes a 378-aa protein with 4 transmembrane domains. It is nearly identical to the Landrace pig STING sequence with 12 nucleotide and 5 amino acid differences. Overexpression of Tibetan porcine STING induced IFN- $\beta$ expression. Future studies will be necessary to investigate whether the difference in STING sequence is related to the difference in disease resistance ability between Landrace and Tibetan pigs.

\section{Acknowledgments}

This work was supported by the Earmarked Fund for Modern Agro-industry Technology Research System of China (CARS-36) and the Scientific Research Fund of Sichuan Provincial Education Department (00924800). We thank Hong Hu, Hong Chen, Li Wang and Hui Diao for help in sample preparation. 


\section{Conflict of Interest}

The authors declare no conflict of interest.

\section{References}

1. Kawai, T.; Akira, S. The roles of TLRs, RLRs and NLRs in pathogen recognition. Int. Immunol. 2009, 21, 317-337.

2. Palm, N.W.; Medzhitov, R. Pattern recognition receptors and control of adaptive immunity. Immunol. Rev. 2009, 227, 221-233.

3. Takeuchi, O.; Akira, S. Innate immunity to virus infection. Immunol. Rev. 2009, 227, 75-86.

4. Akira, S.; Uematsu, S.; Takeuchi, O. Pathogen recognition and innate immunity. Cell 2006, 124, 783-801.

5. Takeuchi, O.; Akira, S. Recognition of viruses by innate immunity. Immunol. Rev. 2007, 220, 214-224.

6. Alexopoulou, L.; Holt, A.C.; Medzhitov, R.; Flavell, R.A. Recognition of double-stranded RNA and activation of NF- $\mathrm{BB}$ by Toll-like receptor 3. Nature 2001, 413, 732-738.

7. Yoneyama, M.; Kikuchi, M.; Natsukawa, T.; Shinobu, N.; Imaizumi, T.; Miyagishi, M.; Taira, K.; Akira, S.; Fujita, T. The RNA helicase RIG-I has an essential function in double-stranded RNA-induced innate antiviral responses. Nat. Immunol. 2004, 5, 730-737.

8. Yoneyama, M.; Kikuchi, M.; Matsumoto, K.; Imaizumi, T.; Miyagishi, M.; Taira, K.; Foy, E.; Loo, Y.M.; Gale, M., Jr.; Akira, S.; Yonehara, S.; Kato, A.; Fujita, T. Shared and unique functions of the DExD/H-box helicases RIG-I, MDA5, and LGP2 in antiviral innate immunity. J. Immunol. 2005, 175, 2851-2858.

9. Nakhaei, P.; Hiscott, J.; Lin, R. STING-ing the antiviral pathway. J. Mol. Cell Biol. 2010, 2, $110-112$.

10. Zeng, W.; Chen, Z.J. MITAgating viral infection. Immunity 2008, 29, 513-515.

11. Ishikawa, H.; Barber, G.N. STING is an endoplasmic reticulum adaptor that facilitates innate immune signaling. Nature 2008, 455, 674-678.

12. Sun, W.; Li, Y.; Chen, L.; Chen, H.; You, F.; Zhou, X.; Zhou, Y.; Zhai, Z.; Chen, D.; Jiang, Z. ERIS, an endoplasmic reticulum IFN stimulator, activates innate immune signaling through dimerization. Proc. Natl. Acad. Sci. USA 2009, 106, 8653-8658.

13. Zhong, B.; Yang, Y.; Li, S.; Wang, Y.Y.; Li, Y.; Diao, F.; Lei, C.; He, X.; Zhang, L.; Tien, P.; Shu, H.B. The adaptor protein MITA links virus-sensing receptors to IRF3 transcription factor activation. Immunity 2008, 29, 538-550.

14. Xie, L.; Liu, M.; Fang, L.; Su, X.; Cai, K.; Wang, D.; Chen, H.; Xiao, S. Molecular cloning and functional characterization of porcine stimulator of interferon genes (STING). Dev. Comp. Immunol. 2010, 34, 847-854.

15. Bäckhed, F.; Ley, R.E.; Sonnenburg, J.L.; Peterson, D.A.; Gordon, J.I. Host-bacterial mutualism in the human intestine. Science 2005, 307, 1915-1920.

16. Shao, L.; Serrano, D.; Mayer, L. The role of epithelial cells in immune regulation in the gut. Semin. Immunol. 2001, 13, 163-175. 
17. Martensen, P.M.; Justesen, J. Small ISGs coming forward. J. Interferon Cytokine Res. 2004, 24, $1-19$.

18. Rhoads, J.M.; Chen, W.; Chu, P.; Berschneider, H.M.; Argenzio, R.A.; Paradiso, A.M. L-Glutamine and $\mathrm{L}$-asparagine stimulate $\mathrm{Na}^{+}-\mathrm{H}^{+}$exchange in porcine jejunal enterocytes. Am. J. Physiol. 1994, 266, G828-G838.

19. Pfaffl, M.W. A new mathematical model for relative quantification in real-time RT-PCR. Nucleic Acids Res. 2001, 29, doi: 10.1093/nar/29.9.e45.

(C) 2012 by the authors; licensee MDPI, Basel, Switzerland. This article is an open access article distributed under the terms and conditions of the Creative Commons Attribution license (http://creativecommons.org/licenses/by/3.0/). 